\title{
Alterations in Epithelial and Mesenchymal Intestinal Gene Expression During Doxorubicin-Induced Mucositis in Mice
}

\author{
Barbara A E de Koning • \\ Dicky J. Lindenbergh-Kortleve • Rob Pieters • \\ Hans A. Büller • Ingrid B. Renes • \\ Alexandra W. C. Einerhand
}

Received: 31 August 2005 / Accepted: 25 January 2006 / Published online: 6 April 2007

(C) Springer Science+Business Media, Inc. 2007

\begin{abstract}
In the current study we aimed to gain insight into epithelial-mesenchymal cross-talk and progenitor compartment modulation during doxorubicin (DOX)-induced mucositis in mice. Intestinal segments were collected on various days after DOX treatment. DOX-induced damage at day 1-2 was characterized by increased epithelial proliferation and apoptosis and a decrease in the expression of epithelial differentiation markers. Concurrently, T-cell factor-4 (TCF4) levels increased and the epithelial differentiation enhancing factor, bone morphogenic protein-4 (BMP4), decreased. During severe damage (day 3), BMP4 levels were significantly increased, which inversely correlated with epithelial proliferation. At the same time, the expression of the epithelial differentiation markers was increasing again. At day 7 , BMP4 levels were down-regulated, while the levels of the epithelial differentiation markers and TCF4 were normalized
\end{abstract}

B. A. E. de Koning $\cdot$ R. Pieters

Department of Pediatrics, Divisions of Pediatric Oncology, Erasmus MC-Sophia Children's Hospital,

Rotterdam, The Netherlands

B. A. E. de Koning - D. J. Lindenbergh-Kortleve · H. A. Büller .

A. W. C. Einerhand

Gastro-enterology and Nutrition, Erasmus MC-Sophia Children's Hospital,

Rotterdam, The Netherlands

I. B. Renes

Neontaology, Erasmus MC-Sophia Children's Hospital,

Rotterdam, The Netherlands

B. A. E. de Koning $(\bowtie)$

Pediatric oncology and gastro-enterology, Erasmus MC-Sophia

Children's Hospital,

Dr Molewaterplein 60,

3015 GJ Rotterdam, The Netherlands

e-mail: b.dekoning@erasmusmc.nl again. These data suggest that in response to DOX-induced damage, BMP4 and TCF4 are modulated in such a way that homeostasis of the progenitor compartment is partly preserved.

Keywords Morphogens - Intestinal differentiation . Epithelial homeostasis · Bone morphogenetic proteins . TCF4

\section{Introduction}

The highly proliferating small intestine is very susceptible to chemotherapy-induced damage [31, 47]. This side effect, often referred to as mucositis, is very painful and can be life threatening due to the enhanced risk of bacterial translocation caused by loss of epithelial barrier function. Each year, around 500,000 patients worldwide suffer from this side effect [7] for which there is still no definitive prophylaxis or treatment.

Drug-induced damage to the highly proliferating stem cells and progenitors located in the crypts of Lieberkühn showed that different classes of cytostatic drugs affect epithelial crypt cells of different topographical and hierarchical status [12, 13, 24]. Methotrexate (MTX), 5-fluorouracil and vincristine for example, induce damage high in the proliferative compartment at crypt position 9-11. Damage induced by MTX is well characterized and shows severe morphological changes characterized by epithelial flattening, villus-atrophy, and specific de-differentiation of enterocytes. The last one is indicated by a decreased expression of the enterocytespecific enzymes but a maintained expression of goblet and Paneth cell-specific genes [29, 38, 41, 45, 46]. Doxorubicin (DOX) is a cytostatic drug that also frequently causes severe 
mucositis. However, DOX-induced damage is not well characterized [28]. DOX preferentially attacks cells at crypt position 4-6, which is just above or at the same level as the intestinal stem cells $[12,32]$. DOX is therefore thought to induce more severe damage, especially to the proliferating compartment at the bottom of the crypts, as for example MTX. In clinical practice, DOX is often used in treatment of solid tumors, leukemia, and lymphomas in both adult and childhood cancer patients $[16,18,21,28]$. Recent studies revealed a dynamic cascade of events leading to chemotherapy-induced mucositis [35]. Not only are the specialized epithelial cells affected by cytostatic-drug treatment, but also the underlying submucosal connective tissue. Under normal physiological circumstances, the epithelium maintains a cross-talk with the mesenchyme. Epithelial-mesenchymal interactions are critical for the normal morphogenesis and maintenance of the crypt-villus axis [15, 23].

Bone morphogenic protein (BMP)- and Wnt-signaling pathways are two of the main pathways involved in embryonic and adult intestinal development [5, 33]. BMPs are morphogenes that constitute a large group of structurally and functionally related proteins of the TGF-beta superfamily. BMP signaling pathways have a key role in organogenesis [2, 37], gastrointestinal development and intestinal homeostasis in adults $[8-11,14,27]$. After intestinal development, BMP4 is exclusively expressed in the intravillus and intracrypt mesenchymal cells, including those adjacent to the intestinal stem cells $[8,10]$. Paracrine BMP signaling occurs specifically in the villus from the mesenchyme to the adjacent epithelium [8], suggesting involvement in epithelial-mesenchymal interactions as a mesenchymal signaling molecule.

The Wnt-signaling pathway also has been implicated in the regulation of the intestinal epithelial proliferation/differentiation balance in vitro [43]. In the intestine of mice deficient for transcription factor TCF4, the main Wnt pathway transcription factor in the intestinal epithelium, loss of proliferative compartments and epithelial cell differentiation are found [17]. Interestingly, the BMP- and Wnt pathways appear to be linked, as was shown by the fact that BMP signaling suppresses Wnt signaling to ensure a balanced control of stem cell proliferation and subsequent epithelial differentiation $[8,10]$.

The objective of this study was to develop an experimental mucositis mouse model to characterize DOX-induced intestinal damage and subsequent repair. In addition, we aimed to correlate the alterations in morphology, epithelial homeostasis, and gene expression with changes in BMP4 and TCF4 expression. This, in order to gain insight into possible modulation of the epithelial-mesenchymal cross-talk and progenitor compartment during chemotherapy-induced intestinal damage and regeneration.

\section{Materials and methods}

Animals

Animal experiments were performed with permission of the Animal Ethics Committee of the Erasmus MC-Sophia. Upon arrival at our institute, 10-week-old male BALB/c mice (Harlan, Horst, The Netherlands) were housed individually during the whole experiment in micro-isolator cages under specific pathogen-free conditions with free access to a standard palletized diet (Hope farms, Woerden, The Netherlands) and water. After 1 week of adjustment to the new environment, the mice were divided into three groups and injected intravenously with doxorubicin (DOX) (Doxorubicin, Pharma Chemie, Haarlem, The Netherlands) on two subsequent days. At day -1 and 0 , the first group of mice was injected with a low dose of DOX of 6 and $4 \mathrm{mg} / \mathrm{kg}$ (low dose) respectively, a second group was injected with a medium dose of 8 and $5 \mathrm{mg} / \mathrm{kg}$ (medium dose) and a third group was injected with a high dose of 10 and $6 \mathrm{mg} / \mathrm{kg}$ (high dose). Controls were given equivalent volumes of $0.9 \% \mathrm{NaCl}$. Mice in the low- and high-dose group were sacrificed at day 1,2, 3 and 7 after the final DOX injection; mice in the medium dose group were only sacrificed at day 3 and 7 . One hour before sacrifice, the mice were injected with $120 \mu 110 \mathrm{mg} / \mathrm{ml}$ 5- Bromo-2'deoxyUridine (BrdU) (Sigma-Aldrich, Zwijndrecht, The Netherlands), an uridine analog, to locate the proliferating cells. Per time point 4-6 DOX-treated animals and 2-4 control animals were sacrificed. Segments of midjejunum were collected and either processed immediately for histological analyses or snap-frozen in liquid nitrogen for storage at $-80^{\circ} \mathrm{C}$ and subsequent protein isolation.

\section{Histochemistry}

Five-millimeter segments of mid-jejunum were fixed in $4 \%$ paraformaldehyde in phosphate-buffered saline (PBS), dehydrated and embedded in Paraplast Plus (Sherwood Medical, Den Bosch, The Netherlands) as previously described [45]. Four $\mu \mathrm{m}$ sections were routinely stained with hematoxylin (Vector Laboratories, Burlingame, CA) and eosin (SigmaAldrich) to study morphological alterations of the crypts and villi. Immunohistochemistry was performed as described previously [45] with some minor modifications. The sections for BrdU staining required an extra adjustment to this protocol of HCL incubation, washing with borate buffer, and pepsin treatment as described before [34]. In short, the sections were blocked as described and incubated overnight with the following antibodies diluted in PBS: to visualize BrdU incorporation, mouse monoclonal anti-BrdU (1:250, Roche Applied Sciences, Indianapolis, IN) was used, as an enterocyte marker rabbit polyclonal anti-rat Sucrase-Isomaltase 
(SI) (1:9000 in PBS, kindly provided by Dr. K.Y. Yeh [49]) was used and as a goblet cell-specific marker rabbit polyclonal anti-rat trefoil factor family (TFF3: 1:3000, kindly provided by Prof. Dr. D.K. Podolsky) was used. Furthermore, BMP expression was visualized with anti-BMP4 (1:100, R\&D Systems, Abingdon, UK). Immunoreactions were detected using Vectastain ABC Elite Kit (Vector Laboratories, Burlingame, CA) and 3,3'-diaminobenzidine tetrahydrochloride (Sigma-Aldrich, Zwijndrecht, The Netherlands).

\section{Crypt and -villus length}

Longitudinal sections of crypts and their corresponding villi were selected so that the base (marked by Paneth cells), middle and top of the crypt were all in the plane of section and thus well orientated. The depth of ten crypts and the length of ten villi were measured on three slides per animal, four animals per time point, with the use of a Nikon Eclipse E800 microscope and IM 500 software.

\section{Protein dot blotting}

The expression of enterocyte markers was detected and quantified as described previously [46]. Briefly, 5-mm segments of the mid-jejunum were homogenized and protein concentrations were measured using the BCA Protein Assay Reagent (Pierce, Rockford, IL) and $50 \mu \mathrm{g}$ protein of each homogenate was dot-blotted on nitrocellulose (Protran BA83, $0.2 \mu \mathrm{m}$; Schleicher \& Schuell, Dassel, Germany). Hereafter, blots were blocked for $1 \mathrm{~h}$ with blocking buffer containing $50 \mathrm{mM}$ Tris, $\mathrm{pH} 7.8,5 \%$ (wt/vol) nonfat dry milk powder (Campina Melkunie, Eindhoven, The Netherlands), $2 \mathrm{mM}$ $\mathrm{CaCl}_{2}, 0.05 \%$ (vol/vol) Nonidet P40 (BDH, Brunschwig Chemie, Amsterdam, The Netherlands) and $0.01 \%$ Antifoam B (Sigma-Aldrich). Blots were incubated overnight at $4{ }^{\circ} \mathrm{C}$ with rabbit polyclonal anti-rat SI (1:1000 [49]), and rabbit polyclonal anti-rat trefoil factor family (TFF3: 1:1500) diluted in blocking buffer. After washing with blocking, the buffer blots were incubated with ${ }^{125}$ I-labeled protein A (specific activity $30 \mathrm{mCi} / \mathrm{mg}$, Amersham Biosciences, Roosendaal, The Netherlands) for $2 \mathrm{~h}$ at room temperature. Specific binding of ${ }^{125}$ I-labeled protein $\mathrm{A}$ to the enterocyte marker antibodies was measured using PhosphorImager detection. The elicited signal was quantified by ImageQuant software (Molecular Dynamics, B\&L systems, Zoetermeer, The Netherlands) and the expression of TFF3 and SI was expressed per $50 \mu \mathrm{g}$ protein of tissue. Average expression levels of TFF3 and SI in the mid-jejunum were calculated per mouse, followed by calculation of the mean expression of TFF3 and SI per time point studied. Subsequently, the average expression of TFF3 and SI of control mice was set at $100 \%$. The specificity of the above-described antibodies was previously confirmed by Western-blot analysis [46].
Western-blot analysis

The same protein homogenate was used as described for protein dot blot analysis. Twenty $\mu \mathrm{g}$ of protein was loaded per lane and run on a $12.5 \%$ SDS-PAGE. The separated proteins were transferred to nitrocellulose membranes (Protran BA83, $0.2 \mu \mathrm{m}$ ) and blocked for $1 \mathrm{~h}$ at room temperature in blocking buffer as described above. The blots were incubated overnight at $4{ }^{\circ} \mathrm{C}$ with primary antibodies diluted in blocking buffer: mouse monoclonal anti-human PCNA, clone PC10 (1:250, Novo Castra Laboratories, Newcastle upon Tyne, UK) and mouse monoclonal anti-human BMP4, clone $3 \mathrm{H} 2$ (1:100), (Novocastra Laboratories, Newcastle upon Tyne, UK), rabbit polyclonal anti-human cleaved Caspase-3 antibody (1:1000, Cell Signaling, Beverly, MA), and mouse monoclonal anti-human TCF-4, clone 6H5-3 (1:250, Upstate, Waltham, MA). After washing with PBS, $0.2 \%$ Tween20 blots bound antibodies were revealed using HRP conjugated goat anti-mouse or rabbit anti-goat secondary antibodies (1:1000) and SuperSignal West Femto Luminol Enhancer kit (Pierce, Rockford, IL). The signal was detected and quantified by the ChemiGenius gel documentation system (Syngene, Cambridge, UK) and the expression of the specific proteins analyzed was expressed per $20 \mu \mathrm{g}$ protein of tissue. Average expression levels of PCNA, BMP4, TCF4, and caspase- 3 in the mid-jejunum were calculated per mouse, followed by calculation of the mean expression of these specific proteins per time point studied. Subsequently, the average expression of PCNA, BMP4, TCF4, and caspase-3 in control mice was set at $100 \%$.

\section{Statistical analysis}

Changes in protein expression levels during damage and regeneration were statistically analyzed using the KruskalWallis H-test and the Mann-Whitney U-test. A $p<0.05$ was considered statistically significant. Data are presented as the mean \pm standard error of the mean (SEM).

\section{Results}

Dose-response analysis of DOX-induced mucositis in an experimental mouse model

To optimize the dose of DOX necessary to induce severe intestinal damage (i.e., villus atrophy, crypt loss and flattening of the epithelial cells) a dose-response curve was performed. Thereto, mice were divided in three treatment groups: low dose, medium dose, and high dose (see Materials and methods, section Animals for details). In the high-dose treatment group, two of the six mice died at day 4. Necropsy showed an excess of fluid in the abdominal cavity of unknown source. Because of the elapsed time after death, morphological evaluation could not be performed. 
Morphological analysis of the low-dose treatment group at days 1 and 2 (data not shown), revealed only a slight increase in crypt length, which became more pronounced at day 3 (Fig. 1B). An increase in crypt length was also seen in the medium dose group at day 3. (Note the medium-dose treatment group was not studied at days 1 and 2). No other morphological changes were seen in the low dose- (days 1-3) and medium-dose group (day 3). In contrast, in the high-dose treatment group we did see severe morphological damage (Fig. 1D-F). Specifically, severe villus atrophy, crypt lengthening, crypt loss, perturbation of crypt arrangement, flattening of crypt and villus epithelium and inflammation were observed at day 3 (Fig. 1F). Moreover, the latter morphological damage was already seen, although in a milder degree, at days 1 and 2 after the high-dose DOX treatment (Fig. 1D, day 1 and Fig. 1E, day 2). At day 7, the intestinal morphology was completely restored in the low-dose and medium-dose group (data not shown), but was still affected in the high-dose group. As in the latter group, the crypt epithelium still showed signs of regeneration, like new crypt formation and less epithelial flattening (Fig. 1G).

Quantitative analysis of crypt and villus length

To quantify the amount of morphological damage induced by the different doses of DOX, the length of the crypts and villi were measured (Fig. 1H-I). In the high-dose group, but not in the low-dose or medium-dose group, a significant increase in crypt length was already observed at day 2 (data not shown). At day 3 the crypt length was significantly increased in each treatment group compared to the control group (Fig. 1H). By day 7 , the length of the crypts had returned to control levels in the low-dose and medium-dose group, but were still increased in the high-dose group. This increase in crypt length, however, showed a trend towards normal levels because the crypt length at day 3 was $41 \%$ above control level but at day 7 this was still only $13 \%$ above control level. The length of the villi did not change in the low-dose or the medium-dose treatment group on each day investigated. However, in the high-dose treatment group the length of the villi were significantly decreased on days $1-3$ and 7 (Fig. 1H, day 3 and 1I, day 7). Because severe intestinal mucositis (day 3) and morphological regeneration (day 7) were seen only in the high-dose treatment group, we continued our studies with the high-dose DOX treatment model.

Effects of high-dose DOX treatment on enterocyte-specific gene expression

To gain insight into the functional capacity of the intestinal epithelium after DOX treatment, a sucrase-isomaltase (SI) immunohistochemical staining was performed. SI is an enterocyte-specific disaccharidase responsible for sucrose degradation. SI is expressed in the brush border of differentiated villus enterocytes directly after weaning [42] and is considered an intestinal epithelial differentiation marker. At day 1 and 2 after DOX treatment SI was expressed in the brush border of enterocytes along the entire villi, comparable to the control epithelium (Fig. 2A, control; B, day 1; C, day 2). At day 3, SI staining showed a patchy pattern with weak staining on less affected parts of the epithelium and even absence of staining on severely damaged epithelial parts of the sections (Fig. 2D). At day 7, during the regenerative phase, the SI staining pattern was comparable to control again (Fig. 2E).

To quantify SI protein expression, protein dot-blot analyses were performed using SI-specific antibodies (Fig. 2F). The SI protein expression level at day 1 was similar to the control situation. At day 2, SI expression levels were significantly decreased and remained significantly decreased at day 3. At day 7, the SI expression levels regained to control levels.

Effects of DOX treatment on goblet cell-specific gene expression

Goblet cell-specific gene expression after high-dose DOX treatment was analyzed by the expression of trefoil factor family 3 (TFF3). TFF3 is a bioactive peptide, produced and secreted by goblet cells, that is involved in epithelial protection and repair $[6,22]$. Immunohistochemical staining of TFF3 showed TFF3 expression by goblet cells in crypts and villi of the jejunum (Fig. 3). At day 1, the TFF3 staining was not visibly altered compared to the control situation (Fig. 3B). At day 2, the immunohistochemical detection of TFF3 decreased visibly, especially in the goblet cells localized in the crypts and lower part of the villi where TFF3 protein could hardly be detected/was absent (Fig. 3C). At day 3, during most severe morphological damage, TFF3 staining by goblet cells in the crypt was reconstituted (Fig. 3D). Day 7 showed complete regeneration of TFF3 (Fig. 3E).

Subsequently, a protein dot-blot analysis was performed (Fig. 3F) using TFF3-specific antibodies to quantify TFF3 levels. At day 1, the TFF3 expression was already significantly decreased to $50 \%$ of control level and maintained at this low level at day 2. At day 3, concomitant with the most severe morphological damage, TFF3 expression returned to control level. TFF3 protein expression was again comparable to control situation at day 7 .

Effect of high-dose DOX treatment on epithelial proliferation

Localization of proliferation was studied by immunohistochemical detection of incorporated BrdU (Fig. 4). In controls (Fig. 4A) BrdU was mainly localized in cells from the 

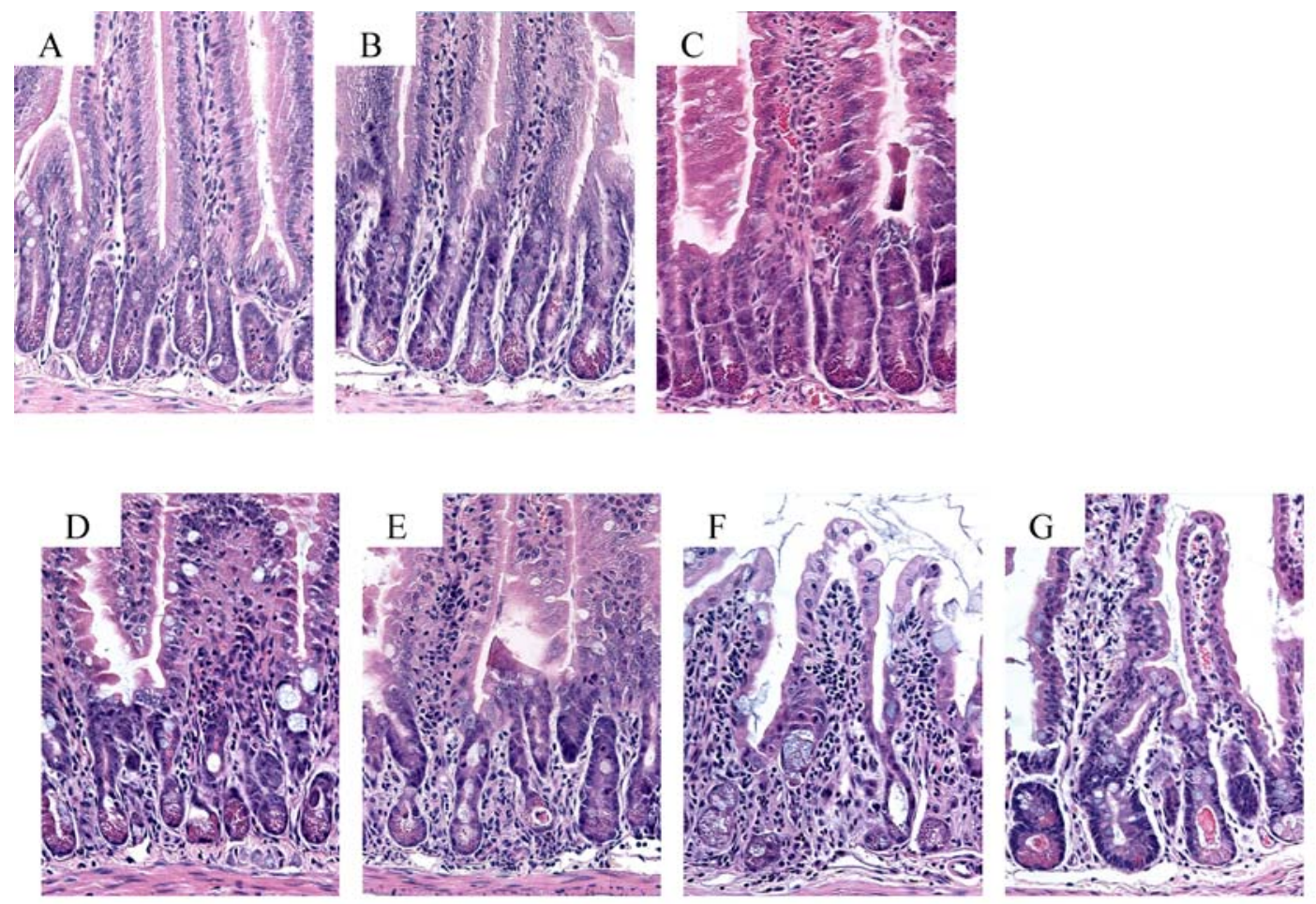

H

Day 3

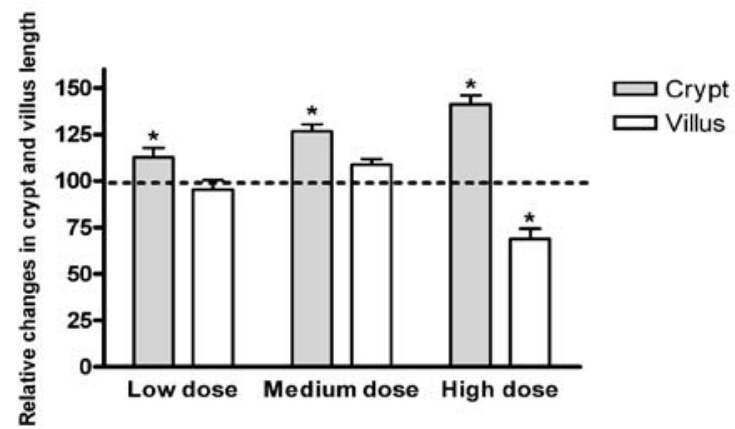

Fig. 1 Morphology of the murine small intestine after low, mediumor high-dose DOX treatment. Morphology of the jejunum of a control mouse (A), at day 3 after low-dose DOX (B) and at day 3 after medium-dose DOX $(\mathbf{C})$, both were mildly affected by DOX treatment. Morphology of the jejunum at day 1 (D) and day 2 (E) after high-dose DOX treatment, which appeared progressively affected and severely deteriorated at day $3(\mathbf{E})$. At day 7 , the regenerative phase, the intestinal morphology of the mice in the low- and medium-dose group was completely restored to control situation (data not shown). The morphology at day 7 of the mice treated in the high-dose group was regenerating, showing new crypt formation but still villus atrophy. These photographs are representative examples of a group of four animals per time-point. To quantify the effect of these different doses of DOX treatment, crypt

bottom of the crypt up to three quarter of the crypt length. At day 1 and 2 the proliferative zone broadened and progressively moved upwards to the crypt-villus junction. At day 3, during severe morphological damage, BrdU-positive cells formed a scattered pattern throughout the remaining crypt
I

Day 7

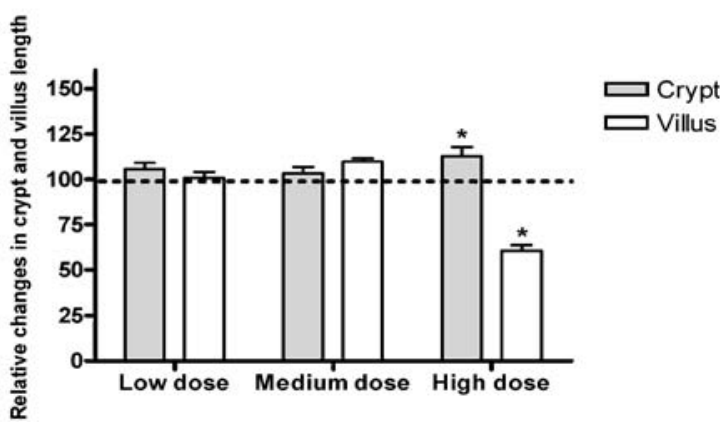

and villus lengths were measured. Crypt and villus lengths of control mice were set at $100 \%$. At day 3 (A), the length of the crypts in all three DOX treatment groups was significantly increased in comparison to the control group. Villus length in the low- and medium-dose group remained at control level. The length of the villi of the high-dose group decreased significantly, indicating significant villus atrophy after high-dose DOX treatment. At day 7 (B) crypt and villus lengths of the low- and medium-dose DOX treatment groups showed complete restoration. The crypts of the high-dose treatment group were still significantly longer, but this increase in crypt length was declining compared to day 3 (day $341 \%$, day 7 13\%). The length of the villi was still significantly decreased at day 7 , showing no signs of regeneration. ${ }^{*} P<0.05$ versus control. The bars are expressed in mean + SEM

structures of the small intestine. In the regenerative phase, at day 7 BrdU-positive cells were located in the lower part of the crypts again.

The changes in proliferation induced by DOX were quantified by Western-blot analysis of PCNA protein expression 

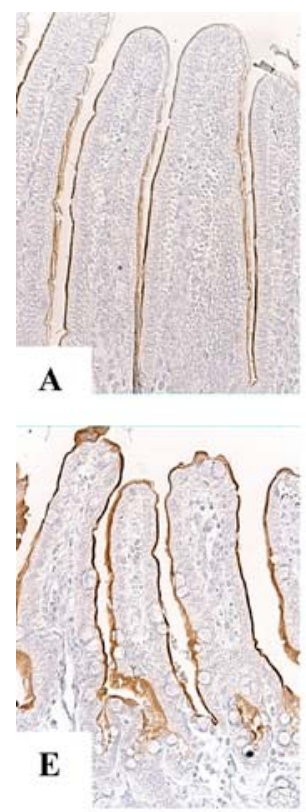
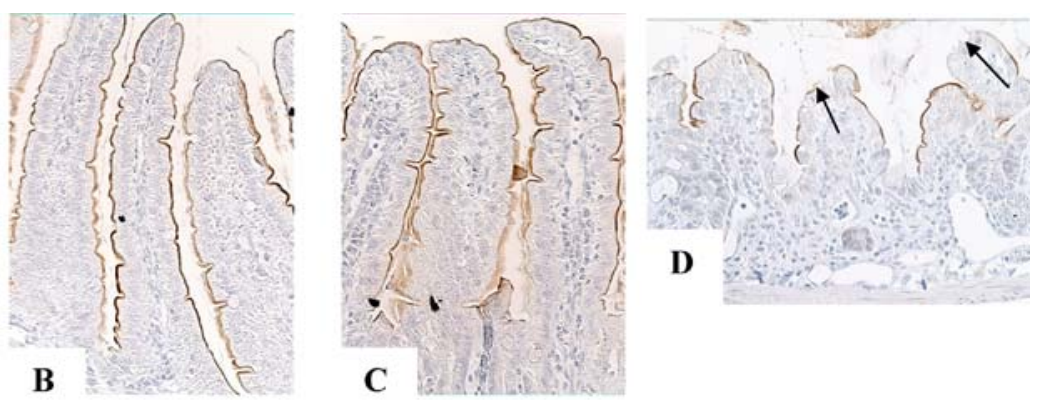

$\mathbf{F}$

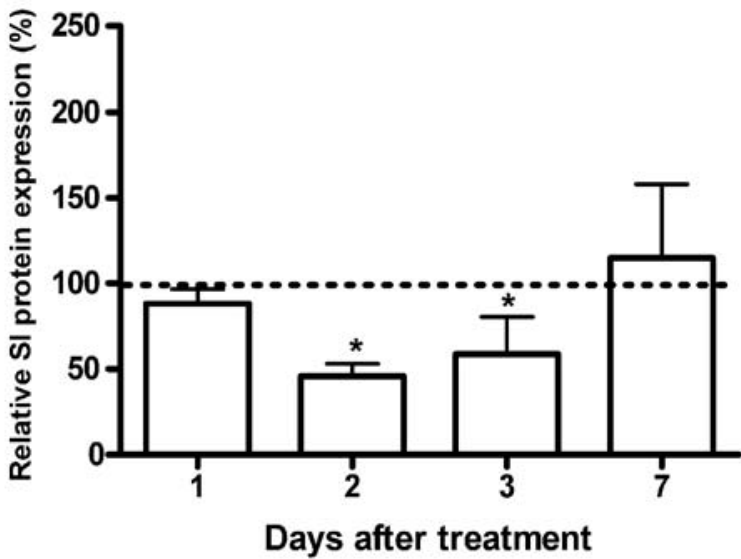

Fig. 2 Effect of high-dose DOX treatment on the enterocyte-specific SI expression in the small intestinal epithelium. SI expression of the jejunum of a control mouse (A), at day 1 (B) and at day $2(\mathbf{C})$. The brush border staining at day 1 and 2 is comparable to control situation. At day 3 (D), SI staining showed a patchy pattern; some parts of the brush border showed SI expression, on some other parts of the brush border SI expression was absent $(\rightarrow)$. SI expression at day 7 (E)

$[4,40]$ using a specific monoclonal PCNA antibody. PCNA protein expression after MTX treatment (Fig. 4F, G) showed a trend of increased expression at days 1 . At day 2, this increased expression was significant compared to control levels, indicating that high DOX treatment did not induce an arrest in proliferation at day 1 and 2. At day 3, PCNA expression was decreased to around $20 \%$ of the normal level and was still $50 \%$ of control level at day 7 . The latter data indicate that the inhibition of epithelial proliferation sustained during the regenerative phase. Overall, the PCNA levels correlated with the immunohistochemical data (Figs. 4A-E vs. $4 \mathrm{G})$

Effect of high-dose DOX treatment on enterocyte apoptosis

Besides proliferation, the influence of DOX treatment on apoptosis was quantified with a polyclonal antibody against cleaved Caspase-3 (Fig. 4F, 5A). Caspase-3 is one of the key executioners of apoptosis [26]. The expression of cleaved Caspase-3 protein was significantly increased at day 1 and showed complete regeneration, and was equal to the control situation. SI protein expression levels were analyzed by dot-blot technique and the SI expression of control mice was set at $100 \%(\mathbf{F})$. The expression of SI remained stable at day 1, but decreased significantly at day 2 and 3 . During the regenerative phase, at day 7 , the SI expression reached control level again. ${ }^{*} P<0.05$ day 2 and 3 versus control. The bars are expressed in mean + SEM

2, at the beginning of DOX-induced morphological damage. At day 3, during severe morphological damage, and day 7 , during regeneration, expression of cleaved caspase- 3 was comparable to control level. Increased apoptosis at day 1 and 2 was primarily located along the crypt axis (Fig. 5B showing day 1 after MTX treatment))

Effect of high-dose DOX treatment on TCF-4 protein expression

To gain insight into the effects of DOX on the epithelial stem cell compartment, the expression of TCF-4 was quantified. TCF-4 is a transcription factor of the Wnt-signaling pathway and is expressed in the gut epithelium in a gradient fashion that is highest at the base of the crypts $[1,19]$. TCF-4 - / mice lose the intestinal epithelial progenitor and stem cell population before crypt formation can be established [17]. Therefore, TCF- 4 has a role in intestinal epithelial stem cell maintenance. The TCF-4 expression (Figs. 4F and 6) remained very stable despite DOX treatment. Only at day 1 the TCF-4 expression increased significantly, although little 

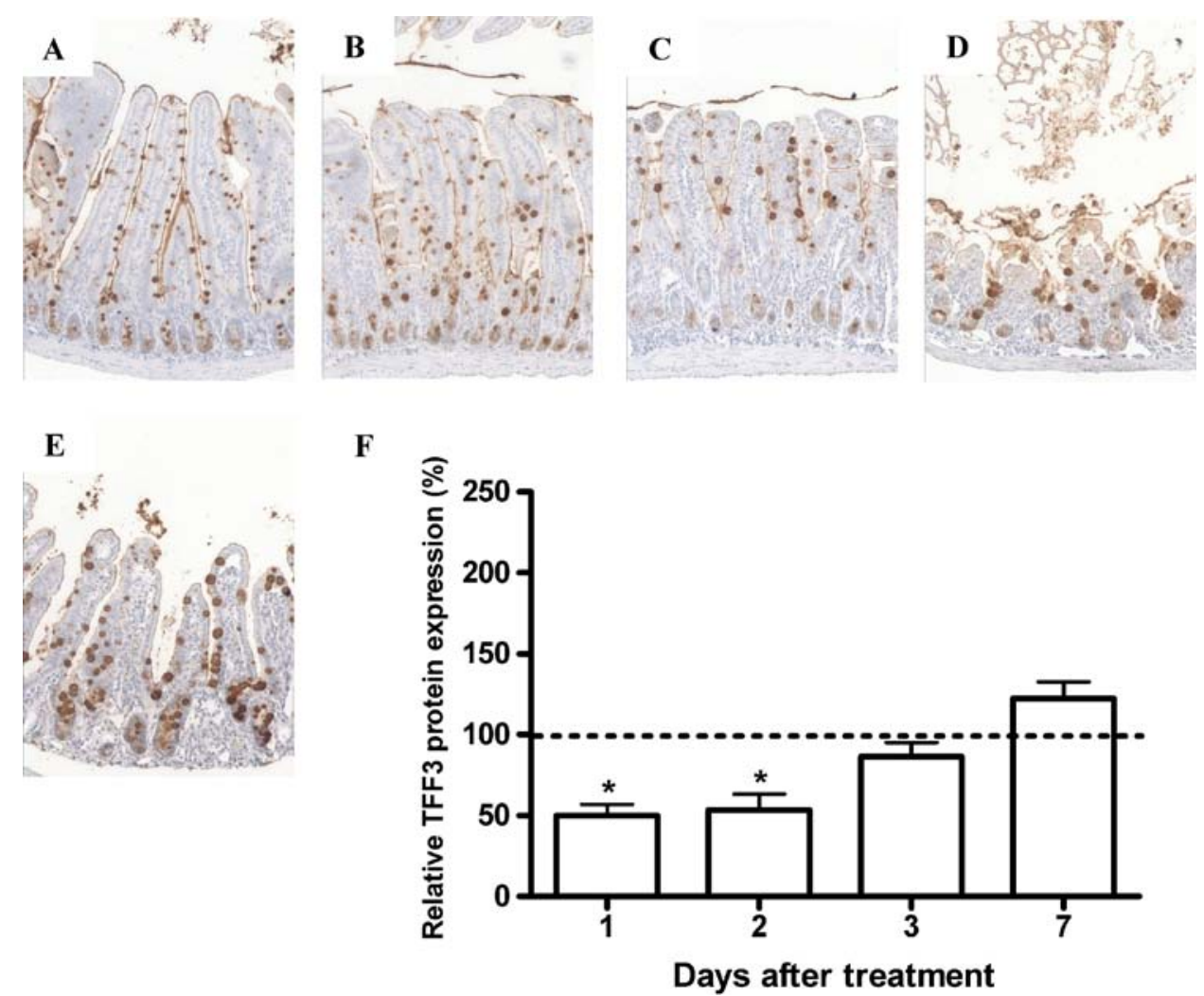

Fig. 3 Effect of high-dose DOX treatment on the goblet-specific TFF3 expression in the small intestine. Immunohistochemical staining of the goblet cell-specific protein TFF3 (A-E). TFF3 expression of the jejunum of a control mouse (A), at day 1 (B), at day $2(\mathbf{C})$, at day 3 (D) and at day 7 (E). In the control situation, TFF3 was expressed in all goblet cells in the crypts and villi, at day $1(\mathbf{B})$, the amount of goblet cells expressing TFF3 maintained at control level. At day $2(\mathbf{C})$, TFF3 expression decreased visibly, the goblet cells localized in the crypts showed no staining. At day 3 (D), during severe morphological damage, TFF3 expression of goblet cells in the crypt was reconsti-

in absolute sense. But at day 2, day 3 and day 7, the TCF4 protein expression remained at control level.

Effect of high-dose DOX treatment on expression of Bone morphogene 4 (BMP4)

To examine the role of BMP4 signaling in intestinal homeostasis the expression of BMP4 was quantified. BMP4 is crucial for epithelial-mesenchymal cross-talk and intestinal homeostasis. BMP4 is expressed in the intravillus and intracrypt mesenchymal cells $[8,10]$, inhibition of BMP signaling causes intestinal architectural abnormalities [8] and decreased differentiation as seen in tumorigenesis [27].

BMP4 protein expression showed a mild decrease in expression level at day 1 after DOX treatment (Figs. 4F, 7A). At day 2, this decrease in BMP4 expression level progressed and was almost significant $(P=0.05)$ in comparison to the control level. Thereafter, BMP4 day 2 but not to control. Day 7 showed a non-significant decrease in expression com- tuted. TFF3 expression at day 7 (E) was regenerated and comparable to control situation. TFF3 protein expression levels were analyzed by dot-blot technique, the TFF3 expression of control mice was set at $100 \%(\mathbf{F})$. TFF3 expression decreased significantly at day 1 and day 2 , the expression was decreased to $50 \%$ of control level. During severe morphological damage at day 3 , the TFF3 expression increased almost to control level. Day 7 showed TFF3 expression even above control level. ${ }^{*} P<0.05$ day 1 and 2 versus control. The bars are expressed in mean + SEM

pared to control mice. BMP4 was predominantly expressed in the intravillus mesenchym as shown by immunohistochemistry (Fig. 7B, C), both in control as in MTX-treated mice.

\section{Discussion}

This study revealed that DOX, in a dose of 10 and $6 \mathrm{mg} / \mathrm{kg}$ induced severe morphological damage to the small intestine of mice within 3 days, which was almost completely regenerated by day 7. Moreover, it revealed that the intestine was virtually not or much less affected by lower doses of DOX. Mucositis induced by the chosen dose of DOX was characterized by an increasing degree of intestinal morphological damage at day 1 and 2, which correlated with a significant increase in both apoptosis and proliferation. During this phase of epithelial hyper-proliferation, the epithelial cells lost their highly differentiated status as measured by a 

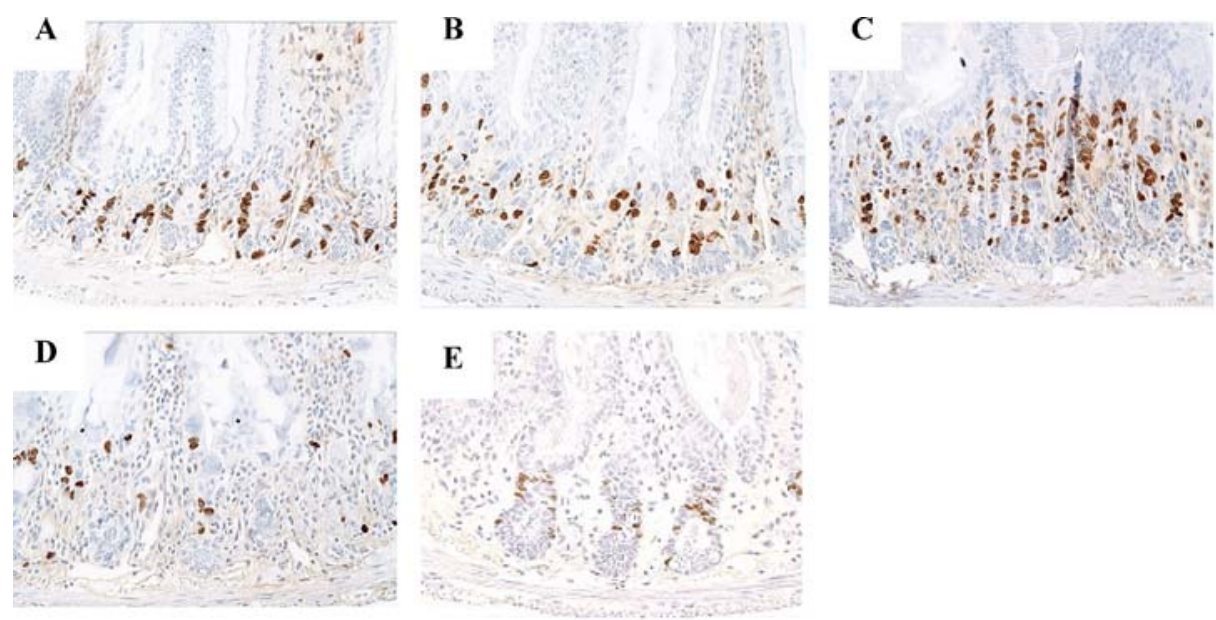

F

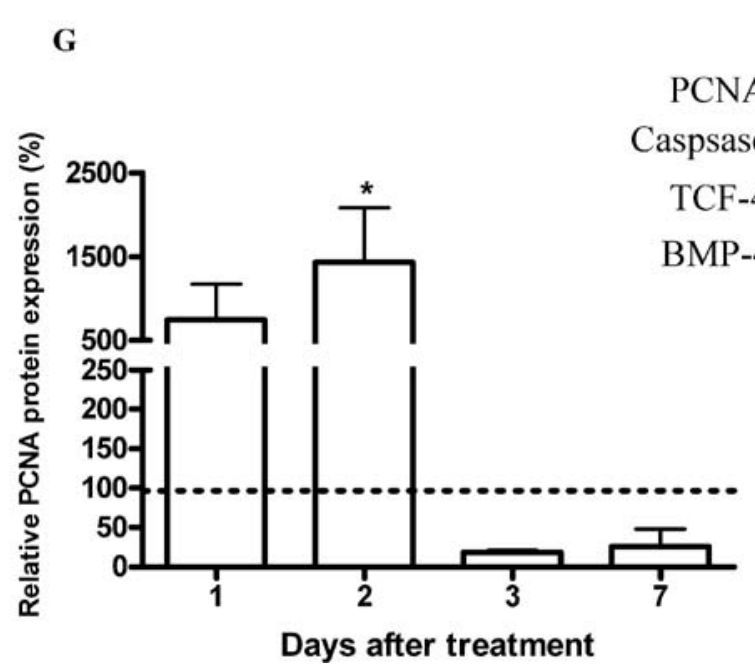

\section{$\begin{array}{lllll}\text { Co } & 1 & 2 & 3 & 7\end{array}$}

CNA …...

Caspsase $17 \mathrm{kd}$

TCF-4 cas $71 \mathrm{kd}$

BMP-4 $21 \mathrm{kd}$

Fig. 4 Effect of high-dose DOX treatment on epithelial proliferation. The localization of epithelial proliferation was visualized by detection of BrdU incorporation. BrdU incorporation of the jejunum of a control mouse (A) and mice at day 1 (B), at day 2 (C), at day 3 (D) and at day $7(\mathbf{E})$. At day 1, the BrdU-positive cells were slightly migrated upwards in the crypt, at day 2 the BrdU-positive cells were seen along the entire length of the crypts. At day 3 the BrdU expression decreased and showed a scattered pattern on some crypts and villi. The location of

significant down-regulation of epithelial-specific SI at days $2-3$. The decreased expression of TFF3 at days 1-2 could be caused by a decrease in goblet cell differentiation, but on the other hand, could also be the result of increased TFF3 secretion. At day 3, the time-point when intestinal damage was most severe, the morphology was characterized by severe villus atrophy, a significant rise in crypt length, epithelial flattening, crypt loss, inhibition of proliferation and impaired epithelial differentiation. During morphological regeneration, at day 7, proliferation started to return to control level, and SI and TFF3 expression levels were normalized again.
BrdU-positive cells at day 7 was confined again to the lower part of the crypts. PCNA, Caspase-3, TCF4 and BMP4 protein expression levels were analyzed by Western-blot technique $(\mathbf{F})$, the PCNA expression of control mice was set at $100 \%(\mathbf{G})$. The expression of PCNA protein showed a non-significant trend of increased expression at day 1 , and a significant increase at day 2. PCNA expression at day 3 and 7 showed a decreasing trend to around $20 \%$ of control level. ${ }^{*} P<0.05$ day 1 and 2 versus control. The bars are expressed in mean + SEM

In order to be able to prevent or treat chemotherapyinduced mucositis, it is essential to know if different cytostatic drugs induce the same or different kinds of intestinal damage. Potten et al. demonstrated that there is a general tendency for antibiotics, like DOX, and radiation to damage the lower cell positions in the crypt near or at the position of the stem cells (position 4-6) [12]. Alkylating agents on the other hand mainly damage cells at position 6-8. Antimetabolites like MTX and a microtubule dissociating agents act on higher cell positions [9-11]. It is, however, unclear if cytostatic drugs attacking at the lowest positions in the crypts cause a different kind of damage than drugs damaging cells at 
Fig. 5 Effect of high-dose DOX treatment on epithelial apoptosis. Caspase- 3 protein expression of the control mice, analyzed by Western-blot technique (Fig 4F), was set at $100 \%$ (A). At day 1 and 2, the Caspase-3 levels increased significantly in comparison to the control levels. At day 3, during severe morphological damage the caspase- 3 expression maintained at control level, the same was seen at day 7 , during the regenerative phase. The increase in apoptosis at day 1 is primarily located in the crypt region $(\mathbf{B}) .{ }^{*} P<0.05$ day 1 and 2 versus control. The bars are expressed in mean + SEM. $\rightarrow$ indicates apoptotic cells

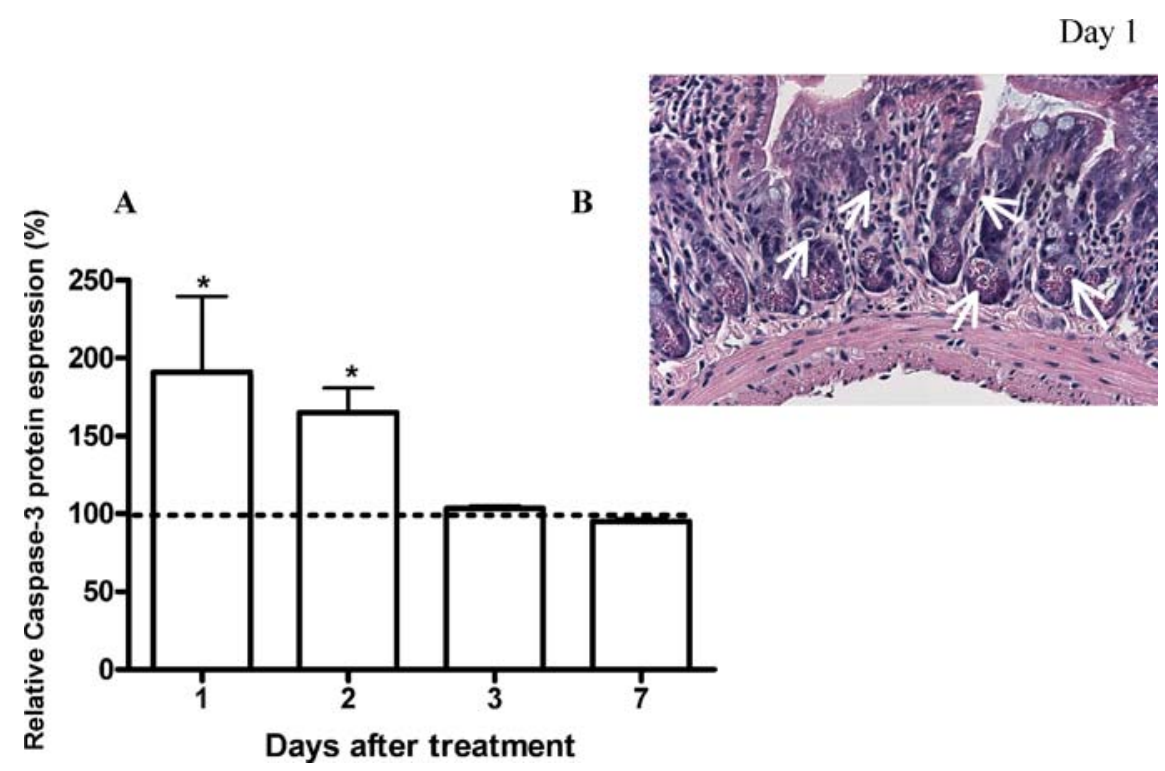

higher positions. If we compare the DOX-induced mucositis as studied presently with the well-characterized MTXinduced mucositis [34, 38, 44-46, 48], then it is clear that there are many similarities and few discrepancies between the two. Although the two drugs affected cells of different hierarchical height [12], they both caused apoptosis, villus atrophy, epithelial flattening, crypt loss and a temporary loss of SI expression and TFF3 expression [45, 46, 48]. Since $\mathrm{SI}$ is involved in carbohydrate metabolism and TFF3 is involved in mucosal repair [6, 22, 30, 42], these data suggest impaired absorption and mucosal repair after DOX as well as after MTX treatment. In contrast, both MTX [45] and DOX hardly affect the expression of lysozyme by Paneth cells in the crypts (data not shown).

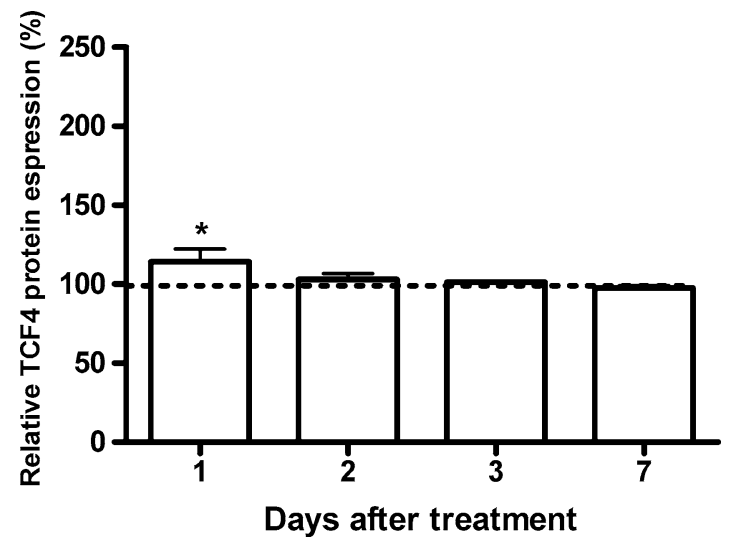

Fig. 6 Effect of high-dose DOX treatment on TCF-4 protein expression. TCF-4 protein expression of control mice, analyzed by Westernblot technique (Fig. 4F), was set at $100 \%$. At day 1, TCF-4 protein expression increased significantly. At day 2 , day 3 , and day 7 , the TCF4 protein expression remained stable at control level. ${ }^{*} P<0.05$ day 1 versus control. The bars are expressed in mean + SEM
Decreased levels of TFF3 after both DOX and MTX was at the same time as epithelial hyper-proliferation [45, 48], but changes in proliferation, induced by MTX treatment followed a different time-line compared to DOX treatment. MTX causes proliferation inhibition within 1 day, followed by a period of hyper-proliferation during severe intestinal damage [34, 45]. DOX treatment leads to immediate hyperproliferation (day 1 and 2) with subsequent inhibition of proliferation during severe morphological damage (day 3 ). Moreover, the cell-fate specific affect of MTX on goblet cells causing goblet cells to accumulate in the crypt and at the top of the villus [45, 46] was not seen after DOX treatment. The reason for these discrepancies remains to be further investigated, but might be directly related to the difference in topographical height (and thus status) of the cells vulnerable to the two different drugs. Overall, however, the similarities in intestinal responses after DOX or MTX treatment are striking. This suggests that there may be common pathways involved in intestinal damage and repair.

Historically, chemotherapy- or radiation-induced mucositis was believed to be solely due to damage to dividing epithelial cells at the bottom of the crypts [20]. However, recently it has become clear that other parts of the intestinal mucosa and submucosa might also be involved $[35,36]$. Here we provide evidence for a mesenchymal contribution to the damage by showing that BMP4, a very important lamina propria derived-morphogen in the small intestine [8, 10], is affected by DOX treatment. BMP4 was modulated by DOX during the onset of damage at days 1 and 2. BMP4 expression decreased almost significant at day 2 , which correlated well with an increasing degree of morphological damage, increased proliferation, and loss of epithelial differentiation as measured by the decreased SI and likely TFF3 expression. Very recently, 
A

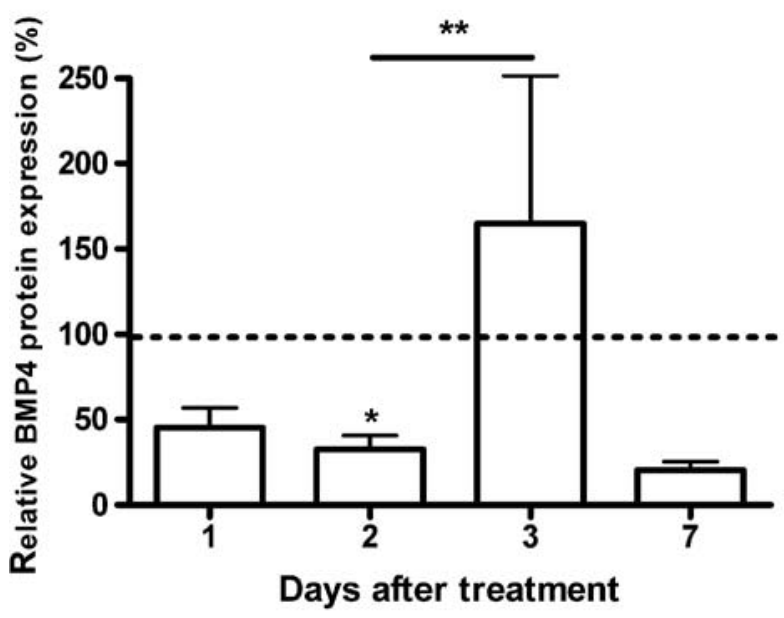

Control

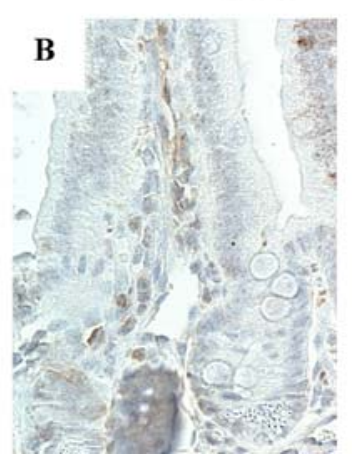

Day 3

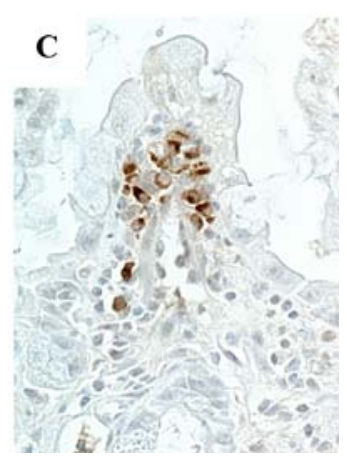

Fig. 7 Effect of high-dose DOX treatment on expression of the morphogen BMP4. The BMP4 protein expression of the control mice, analyzed by Western-blot technique (Fig. 4F), was set at $100 \%$ (A). Expression of the morphogen BMP4 protein showed a decreasing trend at day 1 , which was at the brink of significance $(P=0.05)$ at day 2 in comparison to control expression. At day 3, the BMP4 expression showed an increasing trend. This increase in expression was significant in comparison to day 2. Day 7 showed a decrease in expression again beneath the level of control expression. The localization of BMP4 expression did not change at day three $(\mathbf{C})$ in comparison to control situation $(\mathbf{B}) .{ }^{*} P<0.05$ day 2 versus control, ${ }^{*} P<0.05$ day 2 versus day 3 . The bars are expressed in mean + SEM

a link between the BMP and the Wnt pathways has been demonstrated. It was shown that BMP signaling suppresses Wnt signaling to ensure a balanced control of stem cell proliferation and subsequent epithelial differentiation $[8,10]$. Here we show that there indeed might be a close relationship between BMP and Wnt pathways, because at day 1 when BMP4 expression is decreased, expression of TCF4, a Wnt effector, increased significantly, which correlated well with increased proliferation and inhibited epithelial differentiation. At day 2, BMP4 remained low, whereas TCF4 returned to a normal level and remained at control level during the following days. At day 3, when damage was most severe,

BMP4 was increased, which inversely correlated with proliferation, and correlated with epithelial differentiation, as suggested by the recovery of TFF3 expression level.

BMP is involved in epithelial-mesenchymal signaling [8] and therefore we conclude that the data presented in this study indicate that epithelial-mesenchymal cross-talk is modulated during onset of DOX-induced damage in order to stimulate proliferation instead of differentiation and during severe intestinal damage to induce differentiation and inhibit proliferation. During the regenerative phase, at day 7, BMP4 expression level was down-regulated again, which could be a response to the shortage in number of crypts, since a blockage of BMP4 has been shown to cause stem cells to divide, leading to newly formed crypts $[8,10]$. Therefore, our findings are in line with the roles of the BMP and Wnt/TCF pathway in epithelial homeostasis/morphogenesis. Furthermore, the decrease in BMP4 might also indirectly cause the observed decrease in SI expression, because inhibition of BMP4 stimulates Wnt signaling, and Wnt signaling itself induces $\mathrm{SOX} 9$, a negative regulator of $\mathrm{Cdx} 2$, which is a SI transcriptional activator $[3,10,39]$. In addition, BMP4 has been shown to be directly involved in HNF- $1 \alpha$ expression, also a well-known activator of SI transcription [25]. Thus, the decrease in BMP4 might result in a decrease in $\mathrm{Cdx} 2$ and HNF- $1 \alpha$ expression, two of the most important activators of SI transcription [39]. Currently, it is not known whether the Wnt or BMP4 signaling pathways regulate TFF3 gene expression.

In conclusion, high-dose DOX induces severe damage to the epithelium, which closely resembles damage induced by MTX, indicating that general mechanisms of damage and repair are involved. We show that signaling pathways involving BMP4 and TCF4 and thus epithelial-mesenchymal cross-talk are modulated by DOX-induced damage in such a way that homeostasis of the progenitor compartment is restored by initially inducing cell proliferation and inhibiting differentiation and subsequently inducing differentiation, inhibiting proliferation and promoting crypt fission. Understanding these mechanisms is essential to develop clinical strategies to prevent chemotherapy-induced mucositis.

Acknowledgements We thank Dr. K.Y. Yeh for kindly providing the rabbit polyclonal anti-rat sucrase-isomaltase antiserum and Prof. Dr. D.K. Podolsky for kindly providing the rabbit polyclonal anti-rat trefoil factor family-3 antiserum. This work was supported by a grant from Numico Research BV, Wageningen, the Netherlands and from the Sophia Foundation for Medical Research (SSWO).

\section{References}

1. Barker N, Huls G, Korinek V, et al. (1999) Restricted high-level expression of Tcf-4 protein in intestinal and mammary gland epithelium. Am J Pathol 154:29-35 
2. Bitgood MJ, McMahon AP (1995) Hedgehog and Bmp genes are coexpressed at many diverse sites of cell-cell interaction in the mouse embryo. Dev Biol 172:126-138

3. Blache P, van de Wetering M, Duluc I, et al. (2004) SOX9 is an intestine crypt transcription factor, is regulated by the Wnt pathway, and represses the CDX2 and MUC2 genes. J Cell Biol 166:37-47

4. Bromley M, Rew D, Becciolini A, et al. (1996) A comparison of proliferation markers (BrdUrd, Ki-67, PCNA) determined at each cell position in the crypts of normal human colonic mucosa. Eur $\mathrm{J}$ Histochem 40:89-100

5. de Santa Barbara P, van den Brink GR, Roberts DJ (2003) Development and differentiation of the intestinal epithelium. Cell Mol Life Sci 60:1322-1332

6. Dignass A, Lynch-Devaney K, Kindon H, et al. (1994) Trefoil peptides promote epithelial migration through a transforming growth factor beta-independent pathway. J Clin Invest 94:376-383

7. Duncan M, Grant G (2003) Oral and intestinal mucositis: causes and possible treatments. Aliment Pharmacol Ther 18:853-874

8. Haramis AP, Begthel H, van den Born M, et al. (2004) De novo crypt formation and juvenile polyposis on BMP inhibition in mouse intestine. Science 303:1684-1686

9. Hardwick JC, Van Den Brink GR, Bleuming SA, et al. (2004) Bone morphogenetic protein 2 is expressed by, and acts upon, mature epithelial cells in the colon. Gastroenterology 126:111-121

10. He XC, Zhang J, Tong WG, et al. (2004) BMP signaling inhibits intestinal stem cell self-renewal through suppression of Wnt-betacatenin signaling. Nat Genet 36:1117-1121

11. Hogan BL (1996) Bone morphogenetic proteins in development. Curr Opin Genet Dev 6:432-438

12. Ijiri K, Potten CS (1987) Further studies on the response of intestinal crypt cells of different hierarchical status to eighteen different cytotoxic agents. Br J Cancer 55:113-123

13. Ijiri K, Potten CS (1983) Response of intestinal cells of differing topographical and hierarchical status to ten cytotoxic drugs and five sources of radiation. Br J Cancer 47:175-185

14. Itoh S, Itoh F, Goumans MJ, et al. (2000) Signaling of transforming growth factor-beta family members through Smad proteins. Eur J Biochem 267:6954-6967

15. Kedinger M, Duluc I, Fritsch C, et al. (1998) Intestinal epithelialmesenchymal cell interactions. Ann NY Acad Sci 859:1-17

16. Kelly KM, Hutchinson RJ, Sposto R, et al. (2002) Feasibility of upfront dose-intensive chemotherapy in children with advancedstage Hodgkin's lymphoma: preliminary results from the Children's Cancer Group Study CCG-59704. Ann Oncol 13 Suppl $1: 107-111$

17. Korinek V, Barker N, Moerer P, et al. (1998) Depletion of epithelial stem-cell compartments in the small intestine of mice lacking Tcf4. Nat Genet 19:379-383

18. Kushner BH, Kramer K, LaQuaglia MP, et al. (2004) Reduction from seven to five cycles of intensive induction chemotherapy in children with high-risk neuroblastoma. J Clin Oncol 22:48884892

19. Lee YJ, Swencki B, Shoichet S, et al. (1999) A possible role for the high-mobility group box transcription factor Tcf-4 in vertebrate gut epithelial cell differentiation. J Biol Chem 274:15661572

20. Lockhart PB, Sonis ST (1981) Alterations in the oral mucosa caused by chemotherapeutic agents. A histologic study. J Dermatol Surg Oncol 7:1019-1025

21. Martin M, Garcia-Donas J, Casado A, et al. (2004) Phase II study of pegylated liposomal doxorubicin plus vinorelbine in breast cancer with previous anthracycline exposure. Clin Breast Cancer 5:353357

22. Mashimo H, Wu DC, Podolsky DK, et al. (1996) Impaired defense of intestinal mucosa in mice lacking intestinal trefoil factor. Science 274:262-265
23. Montgomery RK, Mulberg AE, Grand RJ (1999) Development of the human gastrointestinal tract: twenty years of progress. Gastroenterology 116:702-731

24. Moore JV (1985) Clonogenic response of cells of murine intestinal crypts to 12 cytotoxic drugs. Cancer Chemother Pharmacol 15:1115

25. Nastos A, Pogge von Strandmann E, Weber H, et al. (1998) The embryonic expression of the tissue-specific transcription factor HNF1 alpha in Xenopus: rapid activation by HNF4 and delayed induction by mesoderm inducers. Nucleic Acids Res 26:5602-5608

26. Nicholson DW, Ali A, Thornberry NA, et al. (1995) Identification and inhibition of the ICE/CED-3 protease necessary for mammalian apoptosis. Nature 376:37-43

27. Nishanian TG, Kim JS, Foxworth A, et al. (2004) Suppression of tumorigenesis and activation of Wnt signaling by bone morphogenetic protein 4 in human cancer cells. Cancer Biol Ther 3:667-675

28. Patte C, Laplanche A, Bertozzi AI, et al. (2002) Granulocyte colony-stimulating factor in induction treatment of children with non-Hodgkin's lymphoma: a randomized study of the French Society of Pediatric Oncology. J Clin Oncol 20:441-448

29. Pinkerton CR, Cameron CH, Sloan JM, et al. (1982) Jejunal crypt cell abnormalities associated with methotrexate treatment in children with acute lymphoblastic leukaemia. J Clin Pathol 35:12721277

30. Playford RJ, Marchbank T, Chinery R, et al. (1995) Human spasmolytic polypeptide is a cytoprotective agent that stimulates cell migration. Gastroenterology 108:108-116

31. Potten CS, Loeffler M (1990) Stem cells: attributes, cycles, spirals, pitfalls and uncertainties. Lessons for and from the crypt. Development 110:1001-1020

32. Potten CS, Owen G, Booth D (2002) Intestinal stem cells protect their genome by selective segregation of template DNA strands. J Cell Sci 115:2381-2388

33. Radtke F, Clevers H (2005) Self-renewal and cancer of the gut: two sides of a coin. Science 307:1904-1909

34. Renes IB, Verburg M, Bulsing NP, et al. (2002) Protection of the Peyer's patch-associated crypt and villus epithelium against methotrexate-induced damage is based on its distinct regulation of proliferation. J Pathol 198:60-68

35. Sonis ST (2004) The pathobiology of mucositis. Nat Rev Cancer $4: 277-284$

36. Sonis ST, Elting LS, Keefe D, et al. (2004) Perspectives on cancer therapy-induced mucosal injury: pathogenesis, measurement, epidemiology, and consequences for patients. Cancer 100:19952025

37. Sukegawa A, Narita T, Kameda T, et al. (2000) The concentric structure of the developing gut is regulated by Sonic hedgehog derived from endodermal epithelium. Development 127:1971-1980

38. Taminiau JA, Gall DG, Hamilton JR (1980) Response of the rat small-intestine epithelium to methotrexate. Gut 21:486-492

39. Taylor JK, Boll W, Levy T, et al. (1997) Comparison of intestinal phospholipase A/lysophospholipase and sucrase-isomaltase genes suggest a common structure for enterocyte-specific promoters. DNA Cell Biol 16:1419-1428

40. Theon AP, Metzger L, Griffey S (1994) In situ analysis of cellular proliferation in canine, feline and equine tumors by immunohistochemistry: a comparison of bromodeoxyuridine, proliferating cell nuclear antigen, and interchromatin-associated antigen immunostaining techniques. J Vet Diagn Invest 6:453-457

41. Trier JS (1962) Morphologic alterations induced by methotrexate in the mucosa of human proximal intestine. I. Serial observations by light microscopy. Gastroenterology 42:295-305

42. Van Beers EH, Buller HA, Grand RJ, et al. (1995) Intestinal brush border glycohydrolases: structure, function, and development. Crit Rev Biochem Mol Biol 30:197-262 
43. van de Wetering M, Sancho E, Verweij C, et al. (2002) The betacatenin/TCF-4 complex imposes a crypt progenitor phenotype on colorectal cancer cells. Cell 111:241-250

44. Verburg M, Renes IB, Einerhand AW, et al. (2003) Isolation-stress increases small intestinal sensitivity to chemotherapy in rats. Gastroenterology 124:660-671

45. Verburg M, Renes IB, Meijer HP, et al. (2000) Selective sparing of goblet cells and paneth cells in the intestine of methotrexatetreated rats. Am J Physiol Gastrointest Liver Physiol 279:G10371047

46. Verburg M, Renes IB, Van Nispen DJ, et al. (2002) Specific responses in rat small intestinal epithelial mRNA expression and protein levels during chemotherapeutic damage and regeneration. J Histochem Cytochem 50:1525-1536

47. Wright NA, Irwin M (1982) The kinetics of villus cell populations in the mouse small intestine. I. Normal villi: the steady state requirement. Cell Tissue Kinet 15:595-609

48. Xian CJ, Howarth GS, Mardell CE, et al. (1999) Temporal changes in TFF3 expression and jejunal morphology during methotrexateinduced damage and repair. Am J Physiol 277:G785-795

49. Yeh KY, Yeh M, Montgomery RK, et al. (1991) Cortisone and thyroxine modulate intestinal lactase and sucrase mRNA levels and activities in the suckling rat. Biochem Biophys Res Commun 180:174-180 